\title{
Immer mehr Deutsche verzichten auf Behandlungen
}

\begin{abstract}
Aus finanziellen Gründen lehnen mehr und mehr Menschen Eingriffe beim Arzt ab. Deutschland schneidet im EU-Vergleich hier besonders schlecht ab. Mit dem Gesundheitssystem insgesamt zeigten sich die Befragten aber zufrieden.
\end{abstract}

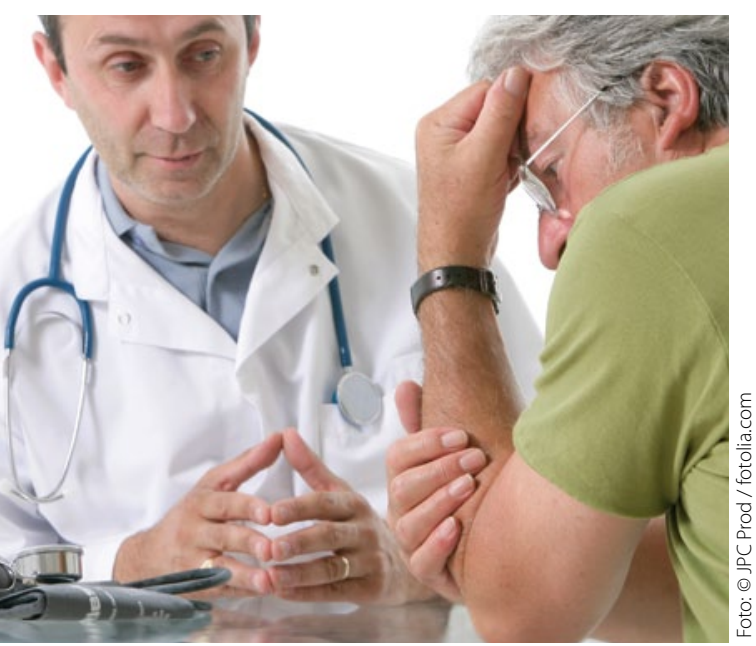

„Kann ich mir die vom Arzt meines Vertrauens empfohlenen Behandlungsmaßnahmen überhaupt leisten?"

- Die Zahl der Deutschen, die aus finanziellen Erwägungen auf medizinische Leistungen verzichten, hat sich innerhalb eines Jahres verdoppelt. Mit 30\% liegt der Anteil der Betroffenen deutlich über dem europäischen Durchschnitt, nur im Nachbarland Polen ist dieser Wert noch größer.

Das zeigt eine Erhebung des Dienstleistungsunternehmens Europ Assistance, das zur italienischen Versiche-

\section{Angehörige brauchen mehr Pflegeberatung}

\begin{abstract}
Hausärzte sollten besser in die Beratung pflegender Angehöriger eingebunden werden, forderten Pflegeexperten. Bislang nutzen nur wenige pflegende Angehörige regelmäßig Beratungsangebote.
\end{abstract}

- Nach Angaben von Ulrich Schneekloth vom TNS Infratest Sozialforschung in München nutzen nur 16\% der pflegenden Angehörigen regelmäßig Beratungsangebote, die Informationsdefizite sind hoch. „Viele sehen einfach keinen Informationsbedarf ", so Schneekloth bei einer Tagung der Ersatzkassen über die „Möglichkeiten und Grenzen ambulanter Versorgung" an der Fachhochschule Frankfurt/Main.

\section{Hausärzte müssen für diese Aufgabe qualifiziert werden}

Oliver Blatt vom Ersatzkassenverband in Berlin wies darauf hin, dass Hausärzte nicht per se Fachleute für das Thema Pflegeversicherung sind. Sie müssten, um die Beratung zu übernehmen, qualifiziert werden: „Als Vertrauenspersonen sind Hausärzte auf jeden Fall eine Institution."

INE . rungsgruppe Generali gehört. Seit 2006 befragt das Unternehmen regelmäßig Erwachsene aus mehreren europäischen Ländern und den USA zu verschiedenen Aspekten des Gesundheitswesens.

Für die Erhebung von Mai und Juni 2012 wurden je 500 Personen aus neun EU-Ländern sowie 1000 Amerikaner befragt. Zwischen 2011 und 2012 stieg demnach der Anteil der Deutschen, die wegen Geldmangels auf medizinische oder zahnmedizinische Behandlungen, Arzneimittel oder Sehhilfen verzichtet hatten, von 15\% auf 30\%. Im EU-Durchschnitt waren es $22 \%$, nach $17 \%$.

Den niedrigsten Anteil verzeichneten Schweden mit vier Prozent (2011: fünf Prozent) und Spanien mit acht Prozent (fünf Prozent).

Auf einer Skala von 1 (schlecht) bis 10 (exzellent) gaben die Deutschen der Organisation ihres Gesundheitswesens die Note 5,5, die einem "gut" entspricht. Nur das österreichische System schnitt mit 6,5 besser ab. Der EU-Durchschnitt betrug 4,7. Die schlechteste Note vergaben die Polen mit einer 2,6.

\section{Ganz zufrieden mit den Ärzten}

Die diagnostischen und therapeutischen Fähigkeiten der behandelnden Ärzte bewerteten die deutschen Befragungsteilnehmer mit einer 5,7, leicht über dem EU-Durchschnitt von 5,6.

Die höchsten Werte erzielten in den Augen ihrer Landsleute die Ärzte in den USA mit einer 7,1, gefolgt von den österreichischen mit 6,5 und den tschechischen mit 6,2.

Im EU-Durchschnitt vertrauten 57\% den Kontrollmechanismen bei Medizinprodukten und 53\% der Kontrolle bei Arzneimitteln. Mit $48 \%$ und $47 \%$ war das Vertrauen der deutschen Befragten etwas geringer.

ISS : 